\title{
Smart technology implementation for road traffic management
}

\author{
Natalia Zhuravleva ${ }^{1}$, Elena Volkova, ${ }^{1, *}$, and Daniil Solovyev ${ }^{2}$ \\ ${ }^{1}$ Emperor Alexander I Petersburg State Transport University, 190031, 9 Moskovsky prospekt, St. Petersburg, Russian Federation \\ ${ }^{2}$ National Research University Higher School of Economics, Faculty of Urban and Regional Development, 101000, 13 Myasnitskaya \\ Ulitsa, Moscow, Russian Federation
}

\begin{abstract}
The escalation of road traffic appears to be a tremendous problem. Various metropolises are influenced by traffic flow congestion and the growth of emissions from petrol usage. In big agglomerations, the expanding quantity of private cars and public transport has caused traffic problems. They have a harmful effect on economy, ecosystem, and on the quality of life in general. It is vital to obtain smart solutions for road traffic management. In this paper, authors propose a way to solve this problem by using smart traffic regulation, which is a part of the bigger smart logistics concept. Agent-based traffic simulation has been chosen to perform this research. This type of modeling is related to the object-oriented way of coding. For modeling and experimental simulation of the intersection in St. Petersburg, AnyLogicmodeling software was used. The results show that proposed algorithm allowed to reduce the average waiting time by $37 \%$. Moreover, the average waiting car number at the intersection has been dropped by 2.5 times after applying the new solution. Thus, projected way of reducing road overload on the selected intersection in St. Petersburg displayed excellent outcomes. However, implementation of the algorithm on other infrastructure objects requires further investigation and analysis.
\end{abstract}

\section{Introduction}

Escalation of road traffic is one of today's challenges. Various metropolises are negatively affected by the traffic flow congestion and the growth of emissions from petrol usage. In big agglomerations, expanding quantity of private, commercial, and public transports has caused traffic problems. In general this problem has a harmful effect on the quality of life, especially in megacities. Thus, it is vital to obtain smart solutions for road traffic management.

"Intelligent Transportation Systems, or ITS, can be defined as the application of computing, information, and communications technologies to the real-time management of vehicles and networks involving the movement of people, goods, and services" [1].

The demand for public transportation in Russia and St. Petersburg in particular, has always been increasing. As information technologies and practices evolve, transportation systems and traffic flows tend to become even more integrated. Therefore, it is crucially important to improve the overall performance of passenger's daily routes by creating effective transportation links with adequate levels of information sharing [2]. The main goal of this research is to investigate how implementation of smart traffic systems can affect street bandwidth of St. Petersburg. To do that, a specific object of the road infrastructure is simulated using a modeling tool.

To achieve the goal, we set and solved several research problems. At first, we have identified current trends in city logistics. Secondly, we have summarized the international experience of adaptive traffic regulation and determined the possibilities of "smart" technologies implementation in urban transport system. Thirdly, we have analyzed the performance indicators of the transport system of St. Petersburg and identified its main problems. Then we had justified the choice of method and technology for simulation modeling. After that we developed a simulation model of the selected intersection in Saint Petersburg. And, finally, we have given some recommendations for improving the efficiency of the urban transport system based on the results of simulation modeling.

\section{City logistics development}

We analyzed some Russian and foreign scientific publications in city logistics and concluded that scientific interest in this area of research is constantly growing. In addition to traditional research problems, such as cargo and passenger transportation, new ideas appear, including smart technologies implementation in cities. [3]

Smart logistics concept includes a set of tools that allows logistics network entities to quickly respond to changing conditions in both external and internal environment. This requires a balanced combination of technology, infrastructure, and management to effectively solve current problems. [4] The growing trend towards urbanization makes smart technologies a

*Corresponding author: moonlight34@ya.ru 
very important tool in city logistics. Urban development management uses a lot of smart technologies to solve different problems.

One of the key tasks of city logistics in megacities is to solve the problem of road network congestion. Some solutions are aimed exactly to solve this issue, including constructing new roads or expanding remaining ones. But there are some problems connected with these solutions. Firstly, construction of new infrastructure facilities is very capital-intensive, and it requires careful planning. Secondly, it often happens that some crucial restrictions must be considered such as geographical and legislative constraints. Smart traffic regulation systems rely on existing road infrastructure and they are aimed to control traffic flows seamlessly. [5] Nowadays, we can already estimate the effectiveness of implementing such solutions using, for example, simulation modeling tools.

\section{The current problem of the transport infrastructure of St. Petersburg}

Countries from all over the world experience numerous problems associated with the usage of vehicles. Ineffective road network [6] has a harmful impact not only on the functionality of the city's transport system, but also on the environment [7]. Of course, some solutions are aimed to solve this issue, including constructing new road networks or expanding remaining ones. However, it is worth noting that such measures have significant drawbacks. Firstly, construction of new infrastructure facilities is very capital-intensive, and it requires careful planning. Secondly, it often happens that some crucial restrictions must be considered such as geographical and legislative constraints. Smart traffic regulation systems rely on existing road infrastructure and they are aimed to control traffic flows seamlessly.

All continuous development plans of St. Petersburg (from 1948 to 2008) inherit key features of the urban environment design. It should be said that some features remained only on paper and were not developed, being cancelled for decades. As a result, there is a strong necessity for the new ways of dealing with drastically increased number of cars (fig. 1).

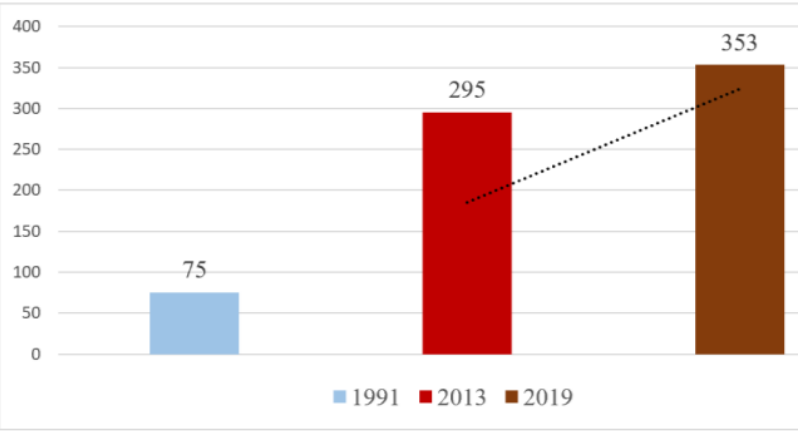

Fig. 1.The number of cars per 1000 citizens in St. Petersburg, Russia. (adapted from: www.gks.ru).

It is worth to mention that the level of motorization in St. Petersburg surpasses the capital's level. Moreover, we may observe an active development of settlements near St. Petersburg, including Devyatkino, Sertolovo and cities in the Leningrad region, closely connected to the Northern capital (Gatchina, Vyborg and others). Based on this, it can be concluded that despite a slight reduction in the number of inhabitants, there is an increase in the average distance covered by the population mainly because of the circular migrations. In other words, we can conclude that the population mobility has amplified in general.

It can be assumed that a significant increase in the number of cars has led to the exhaustion of the capacity of the transport infrastructure. The situation is aggravated by the extremely slow pace of development of alternative modes of transport, such as subway, tram, and bus services. This is especially noticeable during periods of peak load, for example, when the road network gets more snow than usual (fig. 2).

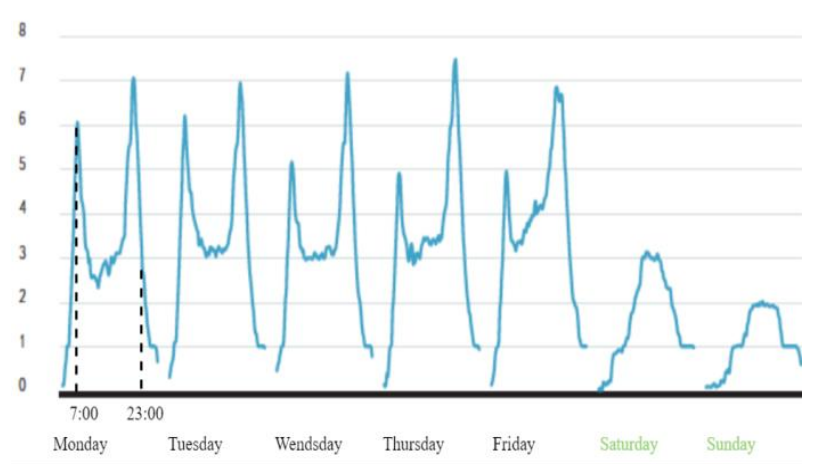

Fig. 2.Average traffic density in St. Petersburg, Russia (adapted from: Yandex.Maps, 2020).

The city center suffers the most from the problem of high workload. This is largely due to the presence of key infrastructure facilities, such as educational organizations, cultural buildings, offices, and shops. Hence, the downtown servers as a main target of circular migrations from other areas.

It is important to note that during the working days the load on transport networks remains relatively constant every day, showing peak values in the morning from 8:00 AM to 10:00 AM and in the evening from about 5:30 PM to 7:30 PM. On weekends, the load on the city's infrastructure is much lower, and on Sunday it shows minimum values of the entire week.

\section{Methods and modeling technology}

Traffic aspects can be defined by measured traffic flows gained through the communication between drivers, cars, and infrastructure [8]. Therefore, in these models researchers are dealing with large amounts of upcoming information: the actions of driver in regard to road conditions, configuration of the streets, travel speed, pedestrian density, location of road signs, etc. By processing these data, it is desirable to increase the total bandwidth. By using the data obtained from several traffic observing sensors, we can develop simulation models. By running an imitation model, we can estimate the overall performance of the road network. 
Based on the analysis of world's practices on using smart traffic regulation systems, we have identified three main groups of them: models based on fuzzy logic, genetic models, and models centered on queue theory. [9] The main advantage of fuzzy logic is flexibility. In this method, you can determine the duration of the traffic light signal not at a specific value, but at a selected interval. Another solution is a genetic algorithm that randomly selects and combines raw data using so-called natural selection. We found out that queuing theory is the simplest, but yet effective method to model traffic flows. Despite their simplicity, models based on queuing theory proved to be the most effective. [10]

Agent-Based Modeling (ABM) can be considered as a modeling style in which individuals, their actions, environment, are clearly represented in a program as agents. This kind of modeling is related to the objectoriented programming approach. In object-oriented programming languages, such as Java, data and programming techniques or processes are compressed in objects that can manage their specific data and interact with other agents. ABM used in this paper, or sometimes called as multi-agent simulation, applies the concept of multi-agent systems in addition to the basic structure of the simulation model. Corresponding simulation mechanisms are applied to active components of the system [11].

\section{Results}

The intersection of Svechnoy Lane with Marat Street was selected. This crossroad is located in the Central District of St. Petersburg. Marat Street is one of the major highways of St. Petersburg. It has a two-way traffic configuration. Svechnoy Lane is a one-way road that runs from BolshayaMoskovskaya Street to LigovskyProspekt. Figure 3 shows a suggested intelligent system configuration at the intersection. According to Google Maps, the average level of congestion in Svechnoy Lane remains in the red zone on weekdays during peak hours, which indicates significant traffic congestion and a substantial decrease in average speed values.

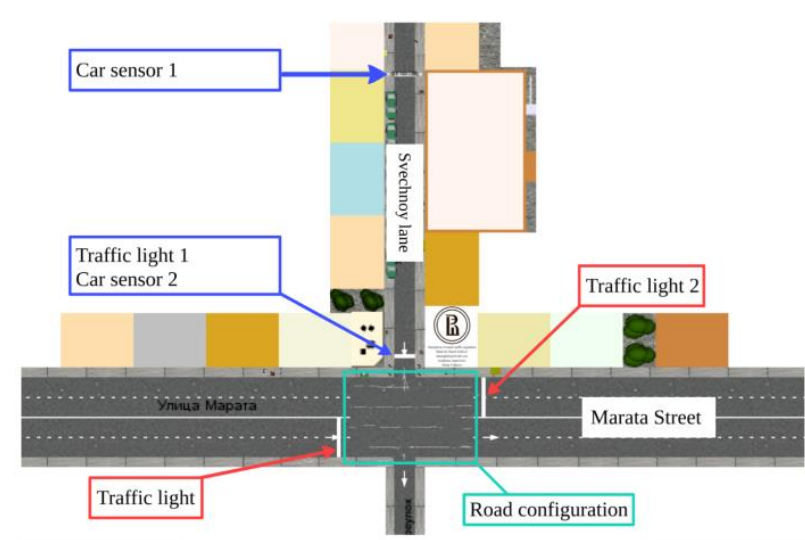

Fig. 3.Suggested ITS configuration at the selected intersection.
At the chosen intersection there are 3 traffic lights that regulate car flows. Traffic light 1 , located on Svechnoy Lane, is controlled by an algorithm that relies on the data from the car sensor 1. An algorithm based on the queuing theory was developed to solve the problem of traffic congestion on Svechnoy Lane. This can be achieved by optimizing traffic light cycles. The values of the traffic lights are taken from the official methodological documents.

For modeling and experimental simulation of the intersection was used AnyLogicmodeling software. This option is defined mainly by accessibility, simplicity, as well as the presence of the road modeling library. Based on agent technology, an algorithm that regulates traffic lights was created (fig. 4).

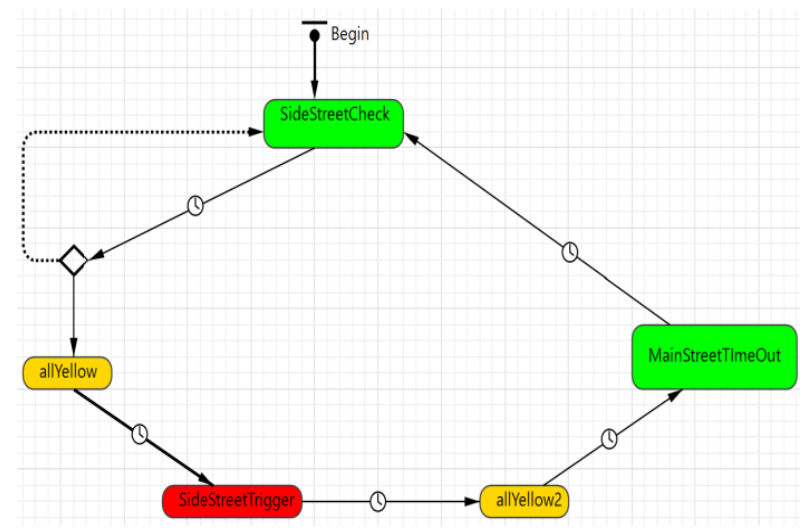

Fig. 4.ITS regulation cycle in AnyLogic model environment.

SideStreetCheck is a loop cycle that updates sensor data every 100 milliseconds. Until the car sensor 1 detects a car, the cycle keeps the following traffic light configuration: traffic light - green signal, traffic light 1 red signal, traffic light 2 - red signal.

When the car sensor 1 detects a car, the allYellow block sets all traffic lights in the system in a yellow signal mode that lasts 3 seconds. Subsequently, the system switches the traffic lights of Marat Street to red signal and opens Svechnoy Lane for 15 seconds.

After that, the allYellow2 block puts 3-second yellow traffic signal again. To avoid abuse of the developed algorithm in cases when the traffic flow from the Svechnoy Lane is unceasing, the block MainStreetTimeOut is added. It is designed to limit the number of locks on Marat Street. Therefore, the number of cycle operations on Svechnoy Lane cannot exceed one time per every 60 seconds. The minimum duration of the green traffic light for Marat Street is 60 seconds, respectively.

\section{Discussion}

The work of the simulation model showed that the implemented algorithm effectively coped with the task of road regulation at the selected intersection. The average number of cars waiting for their traffic signal for the entire experiment was 2 cars which is 2.5 times lower than the current situation (5 cars). The results are presented at fig. 5 . 


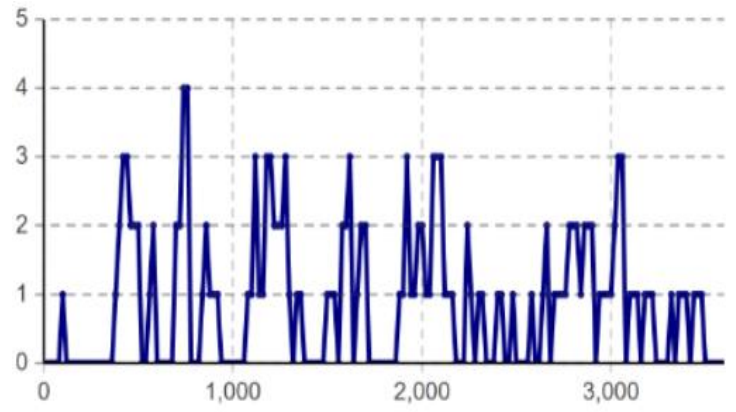

The amount of cars waiting for the green light on Svechnoy lane

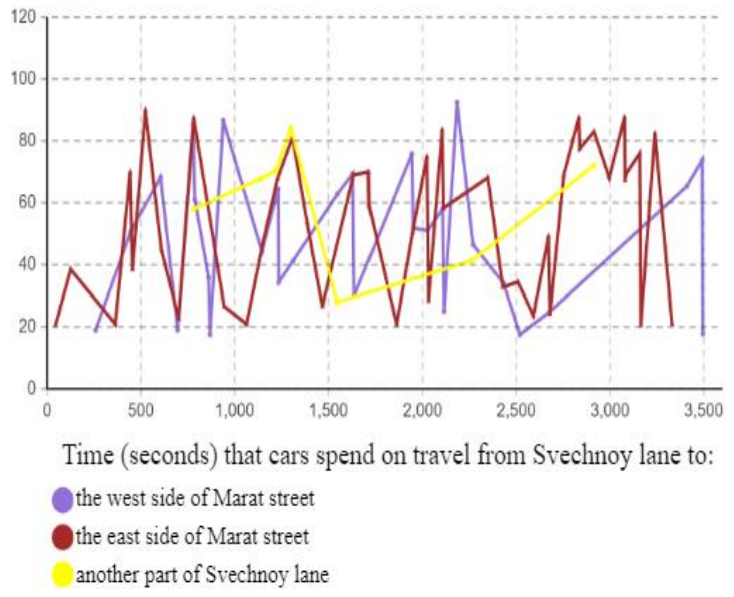

Fig. 5.Model performance data.

In addition to the number of cars in the queue, the average travel time spent by cars performing road maneuvers from Svechnoy Lane was also analyzed (fig. 6).

On average, it took 48.28 seconds for drivers to turn to the west of Marat Street. Averages of 54.40 and 60.16 seconds were determined to turn to the east of Marat Street and to continue moving straight down Svechnoy Lange, respectively (fig. 6). A slight discrepancy in time between the two directions of Marat Street is caused by the different density of traffic flows. Thus, we can assume that in addition to reducing the queue of cars there is also a reduction in the time required to complete moving maneuvers.

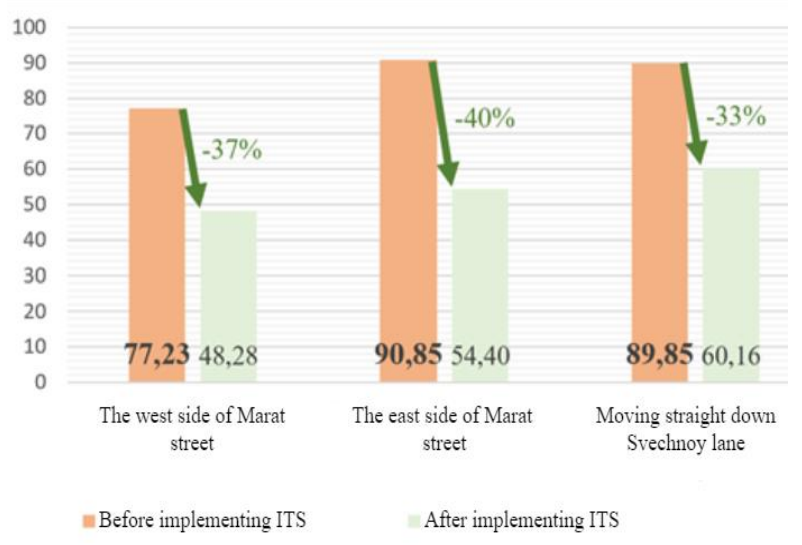

Fig. 6.Comparison of travel time before and after applying ITS (sec).
Installation of ITS is currently taking place in various countries, where it shows impressive results. For example, in Pittsburgh implementation of ITS made it possible to increase the average speed in the city by $25 \%$, as well as reduce air pollution by $20 \%$. Russia is also trying to develop such systems. Most recently, limited testing of the road regulation system that synchronizes traffic lights from different intersection has begun [12]. This once again emphasizes that with proper use city-logistics technologies can certainly help solving road capacity problems.

For further investigation, it is necessary to highlight limitations of the model related to both the software and the availability of empirical data. At first, the duration of the simulation is limited to 1 hour of model time. Secondly, it is not possible to simulate tram lines and pedestrians. Finally, there are limited number of agents in model. Also, we had a lack of empirical data and so decided to make transport flows more consistent. Some of the data was taken from map services.

\section{Conclusion}

Nowadays, it is expected that the transportation processes can be accomplished as securely and swiftly as possible. Studies shown that by modeling real traffic situations, solutions for transport optimization can be found. Numerous traffic models have been created on different levels of abstraction to provide the information of focus in the most transparent way possible.

Agent-based modeling is an approach that allows to capture objects and their interactions. The application of this technology has been done by using the AnyLogic simulation tool. It can be concluded that the proposed algorithm significantly improves the traffic situation by reducing the number of waiting cars on the selected road infrastructure object by 2.5 times: from 5 to 2 . Furthermore, the average travel time was reduced by 37 $\%$.

There are several gaps in our knowledge around some aspects of potential effectiveness of smart traffic regulation systems. Identification of economic effects of these systems can serve as a further area of research. Additionally, the effectiveness of implementing smart traffic regulation in rural areas also requires further analysis.

\section{References}

[1] S. Samadi, A.P. Rad, F.M. Kazemi, H. Jafarian, Journal of Transportation Technologies 2 (2012)

[2] B.M. Lapidus, L.V. Lapidus, The Railway Economics magazine 10 (2016)

[3] S. Hanson, G. Giuliano (ed.), The geography of urban transportation, Guilford Press (2017)

[4] S. Behrendsa, Transportation Research Procedia 12 (2016)

[5] R. Bozzo, A. Conca, F. Marangon, Transportation Research Procedia 3 (2014) 
[6] H.L. Lee, Urbanisation proceeding on unprecedented scale, World Cities Summit, Marina Bay Sands, Singapore (2014)

[7] S. Pelletier, O. Jabali, G. Laporte, Goods distribution with electric vehicles: Review and research perspectives, Montrál, Canada, 2014: Technical Report CIRRELT-2014-44, CIRRELT (2014)

[8] J. Barceló, Fundamentals of traffic simulation (New York, Springer, 2010)

[9] E. Taniguchi, R.G. Thompson, T. Yamada, Procedia-Social and Behavioral Sciences 125 (2014)

[10]F.A. Al-Nasser, H. Rowaihy, IEEE Symposium on Computers \& Informatics (2011)

[11] K.M. Yousef, M.N. Al-Karaki, A.M. Shatnawi, J. Inf. Sci. Eng. 26, 3 (2010)

[12] Pittsburgh cuts travel time by $25 \%$ with smart traffic lights [Electronic resource], Apolitical (14 August 2017) Available at: https://apolitical.co/en/solution_article/pittsburghcuts-travel-time-25-smart-traffic-lights 